\title{
Linx
}

Revue des linguistes de l'université Paris X Nanterre

9 | 1997

Émile Benveniste. Vingt ans après

\section{Benveniste et la question de la fondation d'une syntaxe}

Henri Portine

\section{CpenEdition}

Journals

Édition électronique

URL : http://journals.openedition.org/linx/1002

DOI : 10.4000/linx.1002

ISSN : 2118-9692

Éditeur

Presses universitaires de Paris Nanterre

Édition imprimée

Date de publication : 1 avril 1997

Pagination : 81-94

ISSN : 0246-8743

\section{Référence électronique}

Henri Portine, «Benveniste et la question de la fondation d'une syntaxe », Linx [En ligne], 9| 1997, mis en ligne le 05 juillet 2012, consulté le 19 avril 2019. URL : http://journals.openedition.org/linx/1002 ; DOI : 10.4000/linx.1002 


\title{
Benveniste et la question de la fondation d'une syntaxe
}

\author{
Henri Portine
}

\section{Introduction}

$\mathrm{N}$

ous allons nous interroger sur le rôle et la place de la syntaxe dans l'oeuvre d'Emile Benveniste et plus particulièrement dans les deux tomes des Problèmes de linguistique générale. Pour ce faire, nous serons conduits à distinguer programme syntaxique et projet syntaxique et nous tenterons d'analyser la position de Benveniste par rapport à cette double entrée. Nous ne solliciterons ici que les modèles syntaxiques qui présentent une certaine compatibilité historique avec l'oeuvre de Benveniste.

\section{La syntaxe dans les Problèmes de linguistique générale}

Dans la suite, toute mention ou citation extraite des Problèmes de linguistique générale sera référencée par le numéro du tome et la page. Les autres travaux seront bien évidemment repérés selon la coutume documentaire.

La lecture des deux tomes des Problèmes de linguistique générale conduit le lecteur à s'interroger sur la place faite à la syntaxe. D'une part, une partie semble être clairement consacrée à ce sujet, la quatrième partie, mais sous un titre qui paraît à première vue en limiter la portée: fonctions syntaxiques. D'autre part, le recours à la notion de fonction propositionnelle de Russell 


\section{Henri Portine}

(I:125), l'analyse du génitif comme résultat «d'une transposition d'un syntagme verbal en syntagme nominal» (I:148), la notion de "conversion nominale d'un prédicat» (II : 118), ou encore l'énumération des emplois du pronom moi (II : 199) n'ont pas qu'une connotation syntaxique ; ils intègrent effectivement une véritable dimension syntaxique. Le chapitre $8 \mathrm{du}$ tome II pourrait d'ailleurs se situer sans difficulté dans la quatrième partie. Il y a donc aussi dans l'oeuvre de Benveniste un recours à une interrogation de type syntaxique. Nous distinguerons deux questions :

(a) quelle(s) conception(s) de la syntaxe mobilise(nt) les parties intitulées «fonctions syntaxiques» dans les deux tomes des Problèmes de linguistique générale?

(b) comment et à quelle(s) fin(s) Benveniste convoque-t-il la syntaxe ?

Notons d'abord que, pour Benveniste, «la réflexion sur le langage n'est fructueuse que si elle porte d'abord sur les langues réelles» ${ }^{1}$ (avant-propos du tome 1 des Problèmes). Or cet avant-propos a été rédigé pour la réédition des articles, c'est-à-dire en 1966 ou peu avant. Cette déclaration nous semble donc être une prise de position explicite contre le fait que le mouvement génératif englobait à l'époque en une même recherche les langages artificiels et les langues naturelles, constituant des classes de langages. Dans cette dernière approche, les langues (ou plus exactement les langages sous-jacents aux langues empiriques) étaient considérées avant tout comme des êtres de raison. Cette opposition est primordiale pour le statut de la syntaxe. mais elle ne signifie pas de la part de Benveniste une opposition totale à l'école générativiste : «Par ailleurs on sait que les descriptions formelles des langues ont une utilité directe pour la construction de machines logiques aptes à effectuer des traductions [...].» (I : 30).

\section{Programme et projet syntaxiques}

La perception première donne des formes et l'on sait que ces formes ont pu être envisagées comme les seuls objets sur lesquels un accord quelconque pouvait se faire. Ces formes n'ont pas forcément de propriétés formelles telles qu'elles ont été envisagées au cours du développement de la logique. Il faut donc distinguer la forme en tant qu'objet de perception et la forme en tant qu'élément d'un formalisme. Quel est le statut de la forme des énoncés ?

\footnotetext{
${ }^{1}$ On comparera avec la définition donnée par A. Culioli : «l'étude du langage appréhendé à travers la diversité des langues» (avec, en ajout à la suite, «(et des textes)» dans le numéro VI : 1 de Modèles linguistiques paru en 1984, reprenant une formulation plus lapidaire parue dans «A propos d'opérations intervenant dans le traitement formel des langues naturelles», Mathématiques et sciences humaines,34-1971-p 15.
} 
On a pu considérer les formes d'énoncé comme l'objet fondateur de toute analyse linguistique. C'est penser pouvoir leur faire livrer la clé de l'accès au fonctionnement de l'activité de langage. La forme des énoncés est alors la charpente et il importe d'avoir un programme syntaxique, c'est-à-dire un programme d'analyse proprement fondé sur l'étude des formes d'énoncés.

A cette attitude, l'on peut opposer une conception plus relativisante de la primauté de ces formes. Bien entendu, l'on ne saurait refuser de leur donner un statut : les formes de surface ne sont pas des épiphénomènes. Elles ne sont pas le dépôt aléatoire d'opérations plus fondamentales; elles en constituent plutôt la trace, pour reprendre une formulation de A. Culioli. Dans ce cas, il faut chercher à élaborer un projet syntaxique, c'est-à-dire chercher à doter les analyses d'une dimension syntaxique.

Il convient de cerner d'abord convenablement la notion de programme syntaxique. Nous le ferons à partir de trois cas particulièrement illustratifs :

- le programme génératif,

- le programme dépendanciel,

- le programme que nous nommerons «traditionnel».

\subsection{Le programme génératif²}

Le terme génératif est en fait ambigu. Il désigne - en linguistiqued'une part un mouvement conceptuel, d'autre part une école. Mais comme l'exposé du premier est apparu comme le manifeste de la seconde, la confusion est facile. Reconnaissons que nous n'y échapperons pas totalement. Mais il importe de savoir qu'ici c'est du mouvement conceptuel qu'il sera véritablement question et non de l'école.

Pour bien saisir à la fois l'ampleur et l'objectif du programme génératif, il faut le rapporter à son modèle épistémologique : les machines de Turing. Rappelons rapidement ce dont il s'agit. Les machines de Turing fournissent un procédé de calcul effectif pour l'arithmétique. Une machine de Turing donnée se compose d'une tête de lecture-écriture et d'une bande découpée en cellules qui sert de support à la lecture et à l'écriture. Prenons l'exemple le plus simple : la construction de l'ensemble des nombres naturels en base 10. Lisant la suite des chiffres, la tête de lecture-écriture peut écrire à la suite la suite des entiers naturels. On voit le modèle qui en découle pour l'esprit humain et son application. Ce qui est important pour l'élève de l'école primaire n'est pas de connaître le successeur de 12 ou de 42 mais d'acquérir la capacité de construire le successeur de tout nombre entier, ce qui est a priori formellement analogue à

2 Chomsky (1975) forme sans doute la meilleure introduction à ce programme. Il s'agit de la publication avec introduction d'un ouvrage rédigé en 1955. 


\section{Henri Portine}

la capacité de construire (indéfiniment) l'ensemble infini des nombres dits naturels.

Caractérisons rapidement le programme syntaxique génératif. Il suppose une donnée initiale de type axiomatique, axiome étant à entendre ici dans son sens contemporain, non pas comme une formule postulée dans l'attente de sa démonstration, mais en tant que formule première indémontrable. Il s'agit de la phrase dans sa totalité, représentée par le symbole $S$. A cette donnée initiale s'applique un opérateur (qui peut être considéré comme une tête d'écriture). Cet opérateur peut appliquer indéfiniment les règles permettant de construire l'ensemble infini des phrases de la langue considérée. Tout le problème consiste dans la constitution d'un corps de règles adéquat.

Pour des raisons qui peuvent maintenant paraître évidentes, l'engendrement opère sur des catégories. C'est pourquoi la grammaire générative (et ici il s'agit bien évidemment de l'école), à ses débuts, a donné le sentiment de ne jamais s'intéresser aux fonctions. Selon Chomsky (1975 : 2526), la structure de ce type de programme a été formulée dès 1949. Mais ce n'est bien évidemment que de 1955 à 1965 qu'il s'est trouvé popularisé.

Le programme génératif est un vrai programme syntaxique. Il permet de construire des séquences de mots structurées. Il est autonome : il produit des chaînes terminales (il comporte un système de projection sur la surface). C'est un modèle très tentant : il fournit une solution élégante à la grammaire. Mais il doit être confronté à l'analyse empirique des langues naturelles et c'est là que l'école se sépare du mouvement conceptuel.

\subsection{Le programme dépendanciel ${ }^{3}$}

C'est un programme moins fort que le précédent : il ne produit pas des chaînes terminales ${ }^{4}$, linéaires, mais des structures de relations (nommées stemmas par Tesnière). Chaque élément est mis en relation (en connexion) avec l'élément dont il dépend. Les structures ne sont pas engendrées: elles organisent les éléments de l'énoncé. Les différences entre grammaire de dépendance et grammaire de constituance (c'est-à-dire le programme génératif) caractérisent la différence entre ce programme et le précédent.

Chez Tesnière, le programme dépendanciel est complété par deux types de mises en relation : l'anaphore et la translation. Nous assumons ici la formule complété par. En effet, dans le programme dépendanciel, la relation d'anaphore - qui est une relation à distance tout comme peut l'être la connexion - ne

${ }^{3}$ Cf. Tesnière, 1959.

${ }^{4}$ Cf. B.-N. Grunig (1995) et H. Portine (1995). 
rompt pas la cohérence du fondement. Tel n'est pas le cas au sein du programme génératif : en tant que relation à distance l'anaphore s'oppose à la cohérence fondatrice. Elle ne complète donc pas le dispositif génératif mais s'y ajoute.

La relation de translation (qui reprend la notion de transposition fonctionnelle, cf. Bally, 1932 : 116-127) peut au premier abord paraître un peu surprenante dans la mesure où c'est une relation qui présente une rupture (cf. fig. 1 ci-dessous) : d'un côté, l'on a affaire à une totalité opacifiée quant à sa structure interne (ainsi la proposition complétive de nom est-elle considérée comme un adjectif pour la structure qui l'englobe) ; de l'autre côté l'on a affaire à une structure déployée et donc atomisable en ses moindres éléments (ainsi la proposition complétive de nom est-elle considérée, de façon interne, comme une structure propositionnelle). Cette rupture permet la connexion qui, sans cela, serait rendue impossible par la différence de hiérarchie des éléments en présence (que l'on songe à l'opposition entre «appartenir à» et «être inclus dans» en théorie des ensembles).

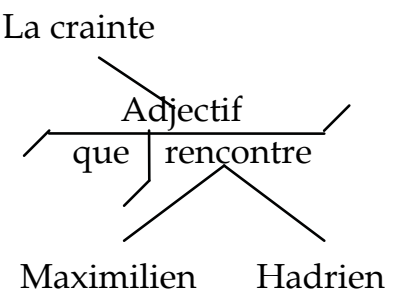

(a)

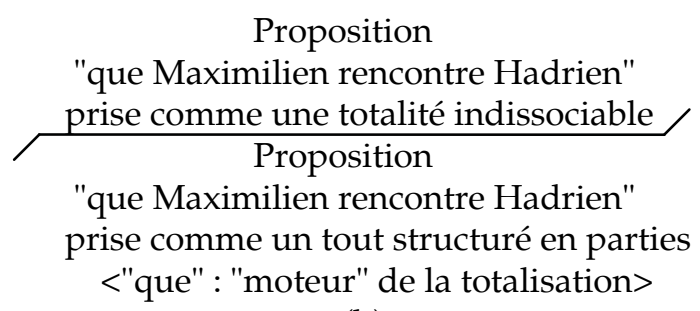

(b)

Figure 1

Le programme dépendanciel est bien un programme syntaxique. Il permet de structurer les énoncés. Mais il ne permet pas d'aboutir à des formes. En ce sens, c'est un programme syntaxique incomplet.

\subsection{Le programme «traditionnel»}

La syntaxe traditionnelle n'a été constituée que pas à pas. Chez les grammairiens grecs la notion de «sujet grammatical», peu utilisée, «est généralement rendue par le terme onoma» (Donnet, $1967: 43-44$ ), conformément à l'analyse qu'a donnée Platon dans le Sophiste (262asq). Il faut rappeler que la morphologie casuelle a longtemps fait obstacle a une distinction relativement claire de la notion de fonction. La Technê grammatikê de Denys le Thrace repose sur une classification et les embryons de mise en évidence de relations liées à la mise en discours chez Apollonius Dyscole (Peri suntaxeôs) ne constituent qu'une ébauche de syntaxe. On peut cependant considérer que s'élabore ainsi un programme syntaxique dont l'expression la 


\section{Henri Portine}

plus nette ne sera donnée que plus tard (voir l'article «Construction» de l'Encyclopédie, dû à Du Marsais, et la partie «Syntaxe» de la Grammaire générale de Beauzée).

S'agit-il véritablement d'un programme syntaxique ? Cette appellation se justifie par la place prise par la syntaxe qui se caractérise alors par les deux opérations présentées comme complémentaires : classer (en «parties du logos» ou en «catégories») et mettre en relation (trouver les «fonctions»). La fonction a une place tardive mais prépondérante. C'est une mise en relation de deux termes, l'un servant de repère à l'autre ${ }^{5}$.

D'un côté, l'on a un véritable programme syntaxique qui repose sur les opérations pré-citées (considérées alors comme cognitivement fondamentales). D'un autre côté, ce n'est pas pour autant un véritable programme syntaxique : la mise en rapport de ces deux opérations avec les chaînes linguistiques effectives a donné lieu à des difficultés qui n'ont pu être surmontées, lorsque cela a été le cas, qu'empiriquement.

\subsection{La notion de projet syntaxique}

A la notion de programme syntaxique nous opposerons celle de projet syntaxique. Il s'agit de marquer la différence entre ce qui se présente comme un mouvement théorisant autonome (programme syntaxique) et la nécessité de relier les analyses de la signification à des configurations (syntaxiques) de sous-chaînes au sein des énoncés.

Nous considérerons qu'il y a projet syntaxique dès lors que les agencements des mots et les rapports possibles entre eux sont considérés comme une donnée à prendre en compte. On s'aperçoit que dès que l'on quitte la notion de programme syntaxique, l'on est conduit à préciser le mode d'intervention de la syntaxe. Nous n'espérons pas résoudre un tel problème ici. Nous voudrions tout simplement tenter de le poser convenablement.

\section{Les conceptions de la syntaxe}

\section{telles qu'elles apparaissent dans les Problèmes}

\subsection{Un dispositif syntaxique de type quasi-génératif?}

La difficulté à déterminer une opposition entre verbe et nom, dès lors que l'on prend la diversité des langues comme une donnée, conduit Benveniste à poser un élément de base subsumant l'opposition verbo-nominale : l'énoncé

\footnotetext{
5 Cette formulation de la notion traditionnelle de fonction permet de la distinguer de celles de Benveniste (cf. plus bas) et de Milner (1989 : 374-375, voir aussi p.275).
} 
assertif fini (I : 154sq). Il caractérise celui-ci selon la forme et selon la fonction. Reprenons chacune de ces caractérisations.

Du point de vue de la forme, «[...] un énoncé assertif fini possède au moins deux caractères formels indépendants: $1^{\circ}$ il est produit entre deux pauses ; $2^{\circ}$ il a une intonation spécifique [...].» Quel est le rôle de ces caractères formels ? Assurément, de doter l'objet à définir du statut d'observable. Les deux critères ont ceci de formel qu'ils supposent une identification de forme soit à l'oreille, soit en laboratoire par le recours à des courbes. On a là une démarche instituant tel type de chaînes linguistiques en phénomène premier.

La date de l'article de Benveniste (1950) interdit tout rapprochement avec l'école nommée "grammaire générative (transformationnelle)». Cependant, l'on peut constater que l'énoncé assertif fini de Benveniste est bien proche de la notion de phrase simple déclarative (Chomsky, 1975 : 15). En outre, il n'appartient pas à «un nombre réduit d'éléments de base» (I:21) mais constitue véritablement un élément premier, bien proche de l'axiome $S$. Il semble y avoir là l'illustration de ce que l'on peut nommer la tentation générativiste: chercher un cadre qui puisse servir de point de départ à l'ensemble des formes constatables. La réécriture que donne Milner de omnis homo mortalis - exemple de Benveniste - sous la forme "[N" ( $\mathrm{N}$ "omnis homo)] [V" (A"mortalis)]» en faisant appel au "phénomène de distorsion» $(1989: 367$, 376) paraît interpréter l'énoncé assertif fini en ce sens.

La seconde caractérisation porte sur la fonction verbale qui peut être assurée ou non par une forme verbale. «Au sein de l'énoncé assertif, la fonction verbale est double: fonction cohésive, qui est d'organiser en une structure complète les éléments de l'énoncé ; fonction assertive, consistant à doter l'énoncé d'un prédicat de réalité.» Commentant la seconde fonction, Benveniste ajoute: «Le contenu de l'énoncé est donné comme conforme à l'ordre des choses.» Il dote ainsi la fonction verbale d'un rôle fondateur.

Cette seconde caractérisation (notamment la fonction cohésive) permet d'hésiter entre d'une part l'équivalence donnée par Milner et rappelée cidessus, et d'autre part une réécriture plus proche de la sémantique générative, du type «Préd. $\left(\operatorname{Arg}_{1}, \ldots, \operatorname{Arg}_{i}\right)$ ». L'on peut rapprocher ce schéma de l'analyse de la composition nominale du sanskrit râja-putrá, «fils de roi»: «être fils n'est pas un prédicat autonome, il exige un autre argument pour se compléter ; ainsi fils, chef, roi valent nécessairement fils-de, chef-de, roi-de» (II : 150). Ce fait de «micro-syntaxe» (II : 145) nous livre une structure, nommée syntaxique par Benveniste (II : 146), qui pourrait très bien expliciter ce qui reste implicite dans l'analyse de la phrase nominale.

L'on trouve peut-être aussi un écho de cette préoccupation fondatrice dans l'emploi de la formule phrase relative (qui apparaît parfois avec des guillemets et parfois sans guillemets et qui est dite terminologie usuelle, I : 212) 


\section{Henri Portine}

qui revendique implicitement le statut phrastique pour la proposition. Certes, nous n'avons pas ici de mécanisme génératif, ni même l'esquisse d'une position transformationnaliste (ce que l'on trouve dans Fondements syntaxiques de la composition nominale: "chaque type de composés est à étudier comme la transformation d'un type d'énoncé syntaxique libre», II : 145-146). Mais Benveniste assume une notion de phrase somme toute assez proche du $S$ (sentence) de la grammaire générative dominant la constitution de la proposition subordonnée relative. «Phrase» dans «phrase relative» a-t-il le même sens que dans «la limite supérieure [de l'intégration] est tracée par la phrase, qui comporte des constituants, mais qui [...] ne peut intégrer aucune unité plus haute» (I : 125) ?

Peut-on échapper à toute tentation générativiste, dès lors que la syntaxe n'est pas le lieu privilégié de l'analyse du langage? Il faut alors éviter de vouloir poser un point de départ syntaxique. De même les notions de source et de transformation deviennent alors suspectes. Il n'y a bien entendu pas de dispositif syntaxique de type génératif chez Benveniste. Mais on trouve quelques formulations qui seraient sans doute à affiner ou à resituer dans un mouvement d'ensemble.

\subsection{Un dispositif syntaxique de type dépendanciel?}

Il apparaît donc que les propositions syntaxiques de Benveniste se situent à côté du programme génératif, y compris dans son étude de la phrase relative. On y trouve d'ailleurs une analyse qui évoque la notion de translation (Tesnière, 1959) constituée dès $1954^{6}$ mais parue deux ans après la publication de l'article de Benveniste.

Reprenons deux points de l'analyse de la phrase relative. D'une part, en ewe, «la phrase relative est obtenue par la conversion d'une phrase verbale en expression nominale au moyen de déterminants pronominaux» (I:210) ; d'autre part, en arabe, «un parallélisme [...] apparaît notamment entre le traitement syntaxique de l'adjectif et celui de la phrase relative» (I : 213). Ces deux fonctionnements se retrouveront en indo-européen.

La conclusion de Benveniste est la suivante : «On a pu montrer [...] que la phrase relative, de quelque manière qu'elle soit rattachée à l'antécédent (par un pronom, une particule, etc.), se comporte comme un adjectif syntaxique déterminé, de même que le pronom relatif joue le rôle d'un article syntaxique déterminatif» (I:222). Commentons la formulation se comporte comme. Elle signifie que - quelle que soit sa structure - le syntagme valant phrase relative

6 Dans «Comment construire une syntaxe» de Tesnière (Bulletin de la Faculté des Lettres de Strasbourg, 1934), la notion de translation n'est pas encore utilisée (cf. les stemmas de la page 224 de cet article et de la page 465 de Tesnière, 1959 qui représentent la même phrase). 
équivaut à un adjectif du point de vue de la structure englobant ce syntagme. On retrouve ici la translation de la relative telle qu'elle est analysée par Tesnière. L'appellation adjectif syntaxique insiste sur la différence entre catégorie adjectivale et fonction adjectivale.

On peut donc trouver chez Benveniste des linéaments de programme syntaxique de type dépendanciel. Cependant, dans son compte rendu des Eléments de syntaxe structurale ${ }^{7}$, Benveniste $(1960: 22)$, émet un avis plutôt réservé : «Il [Tesnière] s'est au fond peu soucié des fondements théoriques de la description et de l'analyse linguistique.» Tout porte à croire qu'il n'a pas cherché à adhérer à un programme de ce type, même si celui-ci fournit des éléments de reformulation de ses analyses.

\subsection{Un dispositif syntaxique de type génétique?}

Un dispositif syntaxique de type génétique affleure aussi dans le traitement de la phrase relative. Benveniste (I : 215-221) oppose deux thèmes pronominaux indo-européens, d'une part (nous remplaçons l'astérisque ambigu parce que pouvant signaler soit une reconstruction soit une séquence impossible - par le signe «+») le thème +yo- (indo-iranien, grec, slave) et d'autre part le thème $+k w o-/+k w i-$ (hittite, latin). Pour chacun des deux thèmes pronominaux, Benveniste évoque la construction de la "phrase relative» avec verbe (pour le thème ${ }^{+} y o-$ ) ou en donne quelques exemples (pour le thème $+k w o-/+k w i-)$ et donne de nombreux exemples de constructions ayant la forme d'un syntagme nominal, par exemple, dans le cas du védique, (nous reprenons la traduction): "Agni, le conquéreur de présents» 8 (I:216), Agni étant à l'accusatif et la détermination nominale par yô au nominatif. Dans le cas où l'on a un syntagme nominal, «le pronom joue le rôle d'un véritable article défini» (I : 216) et +yo- peut être antéposé ou postposé au nom. Benveniste donne aussi quelques exemples de constructions nominales anciennes en latin (I : 220-221). Se constitue ainsi la justification génétique d'une certaine analyse de la relative.

L'intérêt d'un dispositif syntaxique de type génétique se lit dans le texte de Benveniste. Comparer les divers types de "phrase relative» 9 dans leur genèse permet d'éviter projections immotivées et généralisations hâtives. Mais cet intérêt a son revers: constituer des éléments de syntaxe en véritable

\footnotetext{
7 Je remercie M. Dj. Moïnfar de m'avoir signalé ce texte.

8 Bien évidemment, la traduction a perdu ici la trace d'une relative au profit d'une apposition (cf. Agni qui conquiert les présents).

${ }^{9}$ Benveniste semble osciller entre une notion de phrase relative qui recouvrirait des cas de phrase nominale [emplois qui paraissent échapper à la notion de "phrase relative», I : 215] et une mise en cause de la notion de phrase relative [au profit de construction non verbale et de construction verbale, I : 215, ou de forme nominale et de forme verbale, I : 217 ; en opposant syntagme nominal à «phrase relative», ibid.]. La seconde position semble avoir la faveur de Benveniste (cf. I : 218-219).
} 


\section{Henri Portine}

programme syntaxique serait contradictoire car cela reviendrait à ériger l'empirisme en être de raison. Or Benveniste, tout en considérant les langues comme des «organismes empiriques et historiques», n'adopte pas une démarche empiriste: «Si l'on pouvait décrire une à une toutes les morphologies, on constaterait seulement que verbe et nom sont ici distingués, là, non, avec un certain nombre de variétés intermédiaires. Les faits n'enseigneraient pas la raison de cette différence, quand elle se manifeste, ni sa nature.» (I : 154).

\subsection{Un projet syntaxique}

Si l'on ne peut voir dans l'oeuvre de Benveniste un recours à un programme génératif, le modèle dépendanciel ne fournit que des applications locales. Par ailleurs, nous n'avons pas trouvé chez Benveniste de trace d'un programme syntaxique de type «traditionnel». Quant aux éléments de syntaxe de type génétique, ils ne sauraient fournir — nous l'avons vu — les bases d'un programme syntaxique.

L'on n'a donc pas chez Benveniste de programme syntaxique même si quelques formulations - dues sans doute à l'époque de leur écriturepeuvent prêter à illusion. Cela remettrait en cause la constitution d'une partie proprement syntaxique dans les Problèmes si Benveniste n'avait pris la précaution de nommer cette partie «Fonctions syntaxiques». Tout au plus aura$\mathrm{t}$-on un projet syntaxique, c'est-à-dire le souci de tenir compte des modes d'agencements et des formes de surface dans l'analyse.

\section{Une syntaxe ou des éléments syntaxiques? A quelle(s) fin(s)?}

Nous allons maintenant nous interroger sur l'intérêt des questions de syntaxe pour un linguiste qui - comme Benveniste - ne peut adhérer à un programme syntaxique. Mais poser cette question présuppose que l'on traite, au moins rapidement : (a) de l'usage du terme structure chez Benveniste ; (b) de l'opposition entre forme et fonction; (c) de la notion de syntagme.

\subsection{La notion de structure}

Pour Benveniste, le terme structure renvoie à la structure du système. On trouve (I:91-92n.4): «Nous ne tiendrons pas compte de l'emploi non technique du terme structure chez certains linguistes [...]» (voir aussi I : 8-9). Il prétend réserver le terme structure à l'usage technique qu'il en fait alors en l'associant systématiquement à la notion de système : «la notion de structure est étroitement liée à celle de relation à l'intérieur du système» (I : 94). Cela est aujourd'hui banal ainsi que le système en tant que tout organique, ou que les modifications du système au nom de sa cohérence. Nous remarquerons 
néanmoins que Benveniste fait aussi usage (non technique ?) du terme structure à propos de l'énoncé : «structure complète [des] éléments de l'énoncé» (I : 154), «structure syntaxique» (I: 191). Pour Benveniste, la prise en compte d'une dimension syntaxique a son importance : «ce qui donne à la forme le caractère d'une structure est que les parties constituantes remplissent une fonction» (I : 23).

\subsection{Forme et fonction}

Benveniste a insisté à plusieurs reprises sur la différence entre forme et fonction. Les formes sont diverses, variables, alors que la fonction semble correspondre à un invariant (I:155, 158) et représenter l'unicité sous la diversité $^{10}$. Cependant, si l'on se livre à une lecture attentive, et en écartant bien évidemment les emplois relevant de la langue courante, on s'aperçoit que fonction est utilisé dans des contextes rendant son appréhension assez difficile. La fonction verbale assure une fonction cohésive et une fonction assertive (I : 154), on a là trois usages a priori fort différents du mot fonction, le premier attend une définition qui ne vient pas, le deuxième définit un rôle global interne à l'énoncé, et le troisième un rôle externe à l'énoncé, en quelque sorte d'application au réel. La fonction jonctive - difficile à distinguer de la fonction de copule et de la fonction de prédication (I : 189) - est une relation qui lie deux éléments. En est-il de même de la fonction de prédication (sans doute comparable à fonction de détermination, I : 221) ? «La fonction de - $a$ (-la) [il s'agit de l'ewe] est de renvoyer à un terme déjà mentionné dans le discours [...]. En second lieu [...] la postposé à un syntagme verbal prend fonction substantivante» (I : 209) : dans le premier cas, fonction désigne une relation entre deux termes au sein d'un énoncé; dans le second cas, fonction désigne un certain type de catégorisation par rapport à un terme prototypique (cf. aussi fonction d'article, I : 217). Et que penser de : «la fonction du pronom +yo- se définit à la fois par la construction non verbale et par la construction verbale»? On trouve aussi «fonction de composition» (II : 146), «fonctions syntaxiques» (II : 84, 119), «fonction syntagmatique» (II : 120). En revanche, le terme forme désigne constamment les manifestations de surface.

Il est toujours facile de mettre en évidence un emploi paraissant peu assuré d'un terme chez tel ou tel auteur. Aussi n'était-ce pas là notre intention. Le terme fonction est trop récurrent pour ne renvoyer qu'à un mot pratique à utiliser. En fait, les errements apparents de son emploi désignent un problème : celui de la place de la syntaxe justement. Reprenons les emplois du terme fonction. Nous avons un emploi général (à quoi cela sert-il ?), un emploi

10 Cf. «le rapport chaque fois différent entre la fonction syntaxique et les éléments formels qui l'expriment» (I : 208). Comment entendre exactement ( $\mathrm{I}: 50)$, «la linguistique est science des formes exclusivement», à comparer avec I : 126 ? 


\section{Henri Portine}

«russellien» (I : 125, II : 227), et un emploi catégorisant. Sous réserve d'une analyse plus fine, nous proposerons l'essai maladroit de synthèse de la figure 2 pour les deuxième et troisième types d'emplois.

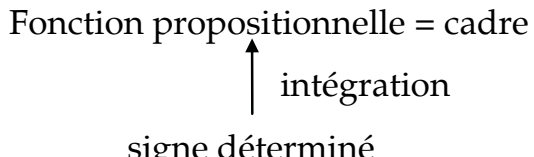

(a)

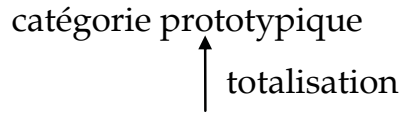

forme déterminée

(b)

Figure 2

\subsection{La notion de syntagme}

La notion de syntagme a donné lieu à deux entreprises totalement divergentes. D'un côté, elle permet de suppléer dans une certaine mesure la syntaxe dans une théorie du signe en fournissant un mode d'agencement des signes en unités plus grandes, une syntagmatique. D'un autre côté, elle fournit un palier intermédiaire aux théories syntaxiques (stricto sensu) : entre le niveau $\mathrm{du}$ lexème et celui de la phrase se situe le niveau des syntagmes remarquables: groupe nominal (le mieux établi), groupe adjectival, groupe verbal (moins assuré), et peut-être d'autres encore (en «GGT», le "groupe prépositionnel», par exemple).

Le second type de manifestation de la notion de syntagme n'apparaît pas véritablement chez Benveniste bien que l'on eût pu l'y attendre dans son texte sur Les niveaux de l'analyse linguistique (1962) qui définit deux niveaux extrêmes, le mérisme et la phrase, et un niveau intermédiaire, le signe ( $:$ : 125). On peut soupçonner l'existence d'un niveau syntagmatique (p.124) au travers de la notion de «mot synnome», mais aussitôt l'affirmation «avec des mots, puis avec des groupes de mots, nous formons des phrases» est mise en doute $\mathrm{du}$ fait de la "progression linéaire» qu'elle suppose. De plus, si l'on trouve «relation syntagmatique» (p.120, 122), «classes syntagmatiques» (p.121), «ordre syntagmatique» (p.122), «éléments syntagmatiques» (p.124), c'est toujours en opposition à «paradigmatique(s)».

L'utilisation du terme syntagme dans les autres textes peut paraître ambiguë. Si «propriété syntagmatique» (II : 97), «oppositions syntagmatiques» (II : 262) ne relèvent que de l'agencement des signes en syntagmes, en revanche la valeur des syntagmes et le fait qu'il s'agisse d'unités à l'intérieur d'une phrase (II : 32), mais aussi l'idée de création (diachronique) d'un syntagme et la notion de syntagme d'auxiliation (II : 128), et peut-être plus encore la «forme spécifique» du syntagme, les notions d'«agencement syntagmatique» ou de «syntagme particulier» (II : 225-227) pourraient faire penser que Benveniste imaginait la possibilité d'un niveau syntagmatique intermédiaire (et donc de syntagmes remarquables). Or l'emploi de syntagmation (II : 228, 229) permet 
d'entrevoir la régulation de cette ambiguïté apparente: il n'y a pas pour Benveniste un niveau spécifique de syntagmes remarquables (il eût sans doute trouvé cela trop rigide), mais une dynamique de l'agencement des signes qui rend possible le passage au niveau de la phrase, celle-ci ne se faisant pas par degrés mais par un saut (l'intégration). Benveniste n'entend pas la formule «niveau intermédiaire» (niveau des signes, I:125) au sens de palier intermédiaire mais au sens de niveau possèdant la double propriété de contenir des constituants et d'être un intégrant, c'est-à-dire un niveau où a lieu une véritable rupture. On a une tri-stratification (là où la grammaire générative a opéré une poly-stratification).

\subsection{Construire une syntaxe}

Benveniste nous désigne un objet à travailler: la constitution d'une syntaxe non comme programme d'explicitation du langage mais en tant que moyen au service de la signification. Cette syntaxe peut-elle être universelle? Il n'est pas sûr que la question elle-même ait un sens.

Critiquant Hjelmslev, Benveniste écrit : «La théorie [de] L. Hjelmslev [...] est une construction d'un "modèle" logique de langue et un corps de définitions plutôt qu'un instrument d'exploration de l'univers linguistique» (I:13). Peut-on constituer une syntaxe qui serait un véritable «instrument d'exploration de l'univers linguistique»?

Mais il faut prendre garde ici au terme instrument. L'on sait que Benveniste s'est élevé contre le fait de voir dans le langage un instrument: «Tous les caractères du langage, sa nature immatérielle, son fonctionnement symbolique, son agencement articulé, le fait qu'il a un contenu, suffisent déjà à rendre suspecte cette assimilation à un instrument, qui tend à dissocier de l'homme la propriété du langage.» (I : 259). La langue n'est pas un instrument au même titre qu'un marteau qui prolonge le bras et accroît le moment de la force. Langue et syntaxe sont inhérentes à l'activité de l'être humain, elles forment un instrument d'exploration de l'univers au même titre que les yeux.

On trouve en 1963 la formule «syntaxe d'énonciation»: «Chaque locuteur ne peut se poser comme sujet qu'en impliquant l'autre, le partenaire qui, doté de la même langue, a en partage le même répertoire de formes, la même syntaxe d'énonciation et la même manière d'organiser le contenu.» (I:25). La syntaxe n'est pas lieu d'engendrement: elle participe de la construction du discours. «C'est dans le discours, actualisé en phrases, que la langue se forme et se configure. Là commence le langage.» (I:131). Et Benveniste ajoute : «nihil est in lingua quod non prius fuerit in oratione», c'est-àdire : rien n'existe dans la langue qui n'ait existé auparavant dans le discours. C'est le discours qui en quelque sorte appelle les cadres syntaxiques qui configurent en retour le discours. Ils ne forment un donné premier que pour 


\section{Henri Portine}

le locuteur qui les trouve déjà là. Ils ne relèvent pas d'un mécanisme d'engendrement mais de formes à la disposition de la production de significations ${ }^{11}$ et constituées dans et par la production de significations ellemême.

\section{Bibliographie}

BALLY, C. (1932) : Linguistique générale et linguistique française. 4ème éd. Berne : A. Francke, 1965

BENVENISTE, E. (1960) : «C. R. de Tesnière, 1959.» BSL, 55 : 2. 20-23

BENVENISTE, E. (1966,1974) : Problèmes de linguistique générale, I et II. Paris : Gallimard

CHOMSKY, N. (1975) : The Logical Structure of Linguistic Theory. New York : Plenum Press

DONNET, D. (1967) : «La place de la syntaxe dans les traités de grammaire grecque des origines au XIIe siècle.» Antiquité classique, 36. 22-46

GRUNIG, B.-N. (1995) : «Instabilité et mutation de la dépendance.» in: F. Madray-Lesigne et J. Richard-Zappella eds: Lucien Tesnière aujourd'hui. Paris: Bibl. de l'Information grammaticale. 113-118

MiLNER, J.-C. (1989) : Introduction à une science du langage. Paris : Seuil

PORTINE, H. (1995) : «Ordre structural et ordre linéaire chez Tesnière : Stemma et arborescence.» in : F. Madray-Lesigne et J. Richard-Zappella eds : Lucien Tesnière aujourd'hui. Paris : Bibl. de l'Information grammaticale. 119-127

TESNIÈRE, L. (1959) : Eléments de syntaxe structurale. Paris : Klincksieck

\footnotetext{
11 Sans faire un parallèle hasardeux, soulignons une certaine analogie avec : «Loin de moi l'idée de minimiser l'importance de la forme syntaxique, mais je me concentrerai presque exclusivement sur la fonction [...].» (J. S. Bruner : Acts of Meaning. Harvard Univ. Press, 1990. Trad. fse : ... Car la culture donne forme à l'esprit. Paris: ESHEL, 1991. p.84), cf. aussi l'importance que Bruner accorde à la notion de signification.
} 